\title{
La morphonologie du roumain : système d'alternances vs système de morphonèmes
}

\author{
Romanian morphonology: \\ system of alternations vs. system of morphonemes
}

\author{
Tomasz Cychnerski \\ Uniwersytet im. Adama Mickiewicza w Poznaniu \\ tomcych@amu.edu.pl
}

\begin{abstract}
The paper confronts two models of the Romanian morphonological system. The first one is a complete set of alternations, which are based on three essential relations of purely formal opposition. These are 83 alternations that are even more subdivided, mainly by the criterion of their direction. The second one is made up of 117 morphonemes derived from directional alternations and is based on qualitative, quantitative and distributional opposition relations. Wider use can be achieved by a system of morphonemes, above all thanks to a strict way of defining the constituent units and their simple representation.
\end{abstract}

Keywords: Romanian morphonology, morphonological oppositions, morphonological system, alternations, morphonemes

\section{INTRODUCTION}

Le but du présent article est de proposer et de confronter deux différents modèles du système morphonologique roumain qui sont à la fois fermés, cohérents et exhaustifs. Bien qu'ils partent tous les deux des mêmes phénomènes morphonologiques de caractère paradigmatique, ils diffèrent par leur degré d'abstraction. Le premier modèle (le système d'alternances) s'arrête aux alternances mêmes, les ordonnant selon certains critères rigoureux de nature purement formelle. Le second modèle (le système de morphonèmes) est à son tour une généralisation du premier et 
s'appuie en plus sur un critère de nature distributionnelle. Cependant, ce critère ne devient effectif qu'après la finalisation du système d'alternances entier, tout en établissant un ensemble ordonné d'unités du second degré d'abstraction - les morphonèmes.

Le système morphonologique proposé est le résultat de nombreuses études détaillées, menées jusqu'à présent par l'auteur dans le domaine de la morphonologie du roumain, qui autorisent pleinement les affirmations présentées ici.

Toutes ces recherches, y compris ce texte-ci, ont employé le système morphologique élaboré pour la langue roumaine par V. Guţu Romalo et le système phonologique développé par E. Vasiliu. Toutefois, les deux conceptions des linguistes roumains ont été partiellement modifiées au fil du temps pour être appliquées à la morphonologie. L'une de ces modifications, la plus importante pour les modèles morphonologiques présentés ici, est un ensemble réduit de phonèmes, composé de [1] sept unités vocaliques $/ \mathrm{i} /, / \mathrm{u} /, / \mathbf{i} /, / \mathrm{a} /, / \mathrm{e} /, / \mathrm{o} /, / \mathrm{o} /$, [2] deux unités transitoires $/ \mathrm{j} /$, /w/, [3] vingt-deux unités consonantiques /m/, /n/, /1/, /r/, /v/, /f/, /z/, /s/, /3/, /s/, /x/, /ts/, /dz/, /tgl, /b/, /p/, /d/, /t/, /J/, /c/, /g/, /k/, toutes définies à l'aide d'un ensemble de quinze traits d'ordre articulatoire \{consonantique, transitoire, haut, bas, antérieur, postérieur, semi-ouvert, nasal, latéral, constrictif, occlusif, labial, apical, palatal, sonore $\}^{1}$.

\section{SYSTÈME D’ALTERNANCES}

Le système proposé comprend toutes les alternances ${ }^{2}$ qui se produisent en roumain et s'appuie sur trois oppositions formelles de base. La première, déterminée par le statut phonologique des membres alternants, permet de distinguer : [1] les alternances non-réductrices - dont aucun membre n'est pas un zéro phonologique, [1'] les alternances réductrices, dont l'un des membres est un zéro phonologique, [2] les alternances consonantiques $(C A)$, dont tous les membres sont des consonnes, [2'] les alternances vocaliques $(V A)$, dont tous les membres sont des voyelles, [2"] les alternances transitoires $(T A)$, dont au moins un membre n'est ni une consonne ni une voyelle. La deuxième opposition emploie comme critère le degré de complexité phonologique des membres alternants et permet de distinguer les alternances monophonémiques, dont chaque membre est un seul et simple phonème, et les alternances biphonémiques, dont au moins un membre est un groupe de phonèmes. La troisième opposition, résultant directement du nombre de membres qui alternent, conduit à la distinction des alternances bimembres, trimembres et quadrimembres. Certaines

\footnotetext{
${ }^{1}$ Sur le même ensemble de traits se base aussi la classification des morphonèmes.

${ }^{2}$ Les prémisses théoriques du système admettent aussi les alternances réversibles telles que $/ \mathrm{t} / \sim \mathrm{t} /$, $/ \mathrm{o} / \sim / \mathrm{o} /, / \mathrm{w} / \sim / \mathrm{w} /$, et cetera.
} 
alternances bimembres peuvent se produire indépendamment et en même temps faire partie d'alternances tri- ou quadrimembres.

Chacune des 83 alternances comprises dans le système peut également être interprétée en tant qu'un processus qui se produit dans la direction du membre morphologiquement le moins marqué au membre morphologiquement le plus marqué. Ce critère permet de spécifier les définitions des alternances comme alternances directionnelles $(d V A, d T A, d C A)$, et conduit souvent à leur classification plus profonde.

Le système d'alternances tenant compte des critères pourvus se présente comme suit :

\section{ALTERNANCES NON-CONSONANTIQUES NON-TRANSITOIRES}

I-1. les alternances non-bimembres trimembres non-monophonémiques non-réductrices :

$V A_{1} \quad$ l'alternance $/ \mathrm{u} / \sim / \mathrm{o} / \sim / \widetilde{\mathrm{oa}} /$ qui se produit $^{3}$

[1] dans la structure du radical flexionnel des verbes du type A PURTA, A JUCA, A DESPUIA, A DUREA, A MURI, A JUPUI, en se réalisant aussi comme l'alternance directionnelle $d V A_{1.1} / \mathbf{u} / \rightarrow\{/ \mathbf{o} /, / \mathbf{o a} /\}^{4}$

[2] dans la structure du radical flexionnel des verbes du type A COASE, en se réalisant alors aussi comme l'alternance directionnelle $\boldsymbol{d} \boldsymbol{V} \boldsymbol{A}_{\mathbf{1 . 2}} / \widehat{\mathbf{o a}} /$ $\rightarrow\{/ \mathbf{o} /, / \mathbf{u} /\}$.

$V A_{2} \quad$ l'alternance $/ \mathrm{e} / \sim / \widehat{\mathrm{ea}} / \sim / \mathrm{o} /$ qui se produit

[1] dans la structure du radical flexionnel du verbe A ÎNVEŞTE, en se réalisant aussi comme l'alternance directionnelle $\boldsymbol{d} \boldsymbol{V} \boldsymbol{A}_{\mathbf{2 . 1}} / \mathrm{e} / \rightarrow\{/ \widehat{\mathrm{ea}} /$, $/ \partial /\}$

[2] dans la structure du radical flexionnel des adjectifs ŞUGUBĂŢ, RĂU, en se réalisant alors aussi comme l'alternance directionnelle $\boldsymbol{d} \boldsymbol{V} \boldsymbol{A}_{\mathbf{2 . 2}} / \mathrm{\partial} / \rightarrow$ $\{/ \mathbf{e} /, \widehat{\mathbf{e a}} /\}$.

I-2. les alternances non-bimembres trimembres non-monophonémiques réductrices :

$V A_{3}$ l'alternance $/ \mathrm{e} / \sim \widehat{\mathrm{ea}} / \sim \varnothing$ qui se produit dans la structure du radical flexionnel des verbes du type A PĂREA, A MÂNEA, A VEDEA, A TĂCEA, en se réalisant aussi comme l'alternance directionnelle $d V A_{3} / \mathbf{e} / \rightarrow\{\widehat{/ e a} /, \boldsymbol{\emptyset}\}$.

I-3. les alternances non-bimembres trimembres monophonémiques non-réductrices :

$V A_{4}$ l'alternance $/ \mathbf{i} / \sim / \mathrm{a} / \sim / \mathrm{a} /$ qui se produit dans la structure du radical flexionnel des verbes du type A RĂMÂNE et A MÂNEA, en se réalisant aussi comme l'alternance directionnelle $\boldsymbol{d} \boldsymbol{V} \boldsymbol{A}_{4} / \mathbf{i} / \rightarrow\{/ \mathbf{\partial} /, / \mathbf{a} /\}$.

\footnotetext{
${ }^{3}$ Pour des raisons d'économie de l'argumentation, les contextes définitoires des membres individuels constitutifs des alternances seront omis.

${ }^{4} \mathrm{La}$ flèche indique la direction de l'alternance. Entre parenthèses est pris l'ensemble de membres marqués, avec lesquels alterne séparément et tour à tour le membre non-marqué.
} 
$V A_{5} \quad$ l'alternance $/ \mathrm{a} / \sim / \mathrm{e} / \sim / \mathrm{a} /$ qui se produit

[1] dans la structure du radical flexionnel des verbes du type A ÎNVĂŢA, A ÎMBĂTA, A APĂSA, en se réalisant aussi comme l'alternance directionnelle $d V A_{5.1} / \mathbf{a} / \rightarrow\{/ \mathbf{e} /, / \mathbf{a} /\}$

[2] dans la structure du radical flexionnel des verbes du type A VEDEA, en se réalisant alors aussi comme l'alternance directionnelle $d \boldsymbol{V} \boldsymbol{A}_{5.2} / \mathrm{e} / \rightarrow$ $\{/ \mathbf{a} /, / \mathbf{a} /\}$.

I-4. les alternances non-bimembres trimembres monophonémiques réductrices :

$V A_{6} \quad$ l'alternance $/ \mathrm{a} / \sim / \mathrm{e} / \sim \varnothing$ qui se produit dans la structure du radical flexionnel des verbes du type A DEOCHEA, A DESFOIA, A ÎNTÂRZIA, A VEGHEA, A ÎNTEMEIA, A STUDIA, en se réalisant aussi comme l'alternance directionnelle $\boldsymbol{d} V \boldsymbol{A}_{\mathbf{6}} / \mathbf{a} / \rightarrow\{/ \mathbf{e} / \sim \emptyset\}$.

$V A_{7} \quad$ l'alternance $/ \mathrm{a} / \sim / \mathrm{\partial} / \sim \varnothing$ qui se produit dans la structure du radical flexionnel des verbes du type A OCUPA, A ARUNCA, A UMBLA, A CONTINUA, A LUCRA, A PARCA, A PIUA, A EFECTUA, en se réalisant aussi comme l'alternance directionnelle $d V A_{7} / \mathbf{a} / \rightarrow\{/ \mathbf{\partial} /, \boldsymbol{\emptyset}\}$.

I-5. les alternances bimembres non-monophonémiques non-réductrices :

$V A_{8} \quad$ l'alternance $/ \mathrm{e} / \sim$ /ea/ qui se produit

[1] dans la structure du radical flexionnel des verbes du type A ÎNTREBA, A LUCRA, A TRECE, A ALEGE, A REPEZI, A IUBI, et des adjectifs du type MIŞEL, GREU, MEU, en se réalisant aussi comme l'alternance directionnelle $d V A_{8.1}(/ \mathrm{e} / \rightarrow / \overline{\mathrm{ea}} /)_{1}$;

[2] dans la structure du radical flexionnel du verbe A FREMĂTA, en se réalisant dans ce cas aussi comme l'alternance directionnelle $\boldsymbol{d} \boldsymbol{V} \boldsymbol{A}_{\mathbf{8 . 2}}$ $(/ \mathrm{e} / \rightarrow \widehat{\mathrm{ea}} /)_{2}$;

[3] dans la structure du radical flexionnel du verbe A LEGĂNA, en se réalisant dans ce cas aussi comme l'alternance directionnelle $\boldsymbol{d} \boldsymbol{V} \boldsymbol{A}_{\mathbf{8 . 3}}$ $(/ \mathrm{e} / \rightarrow \widehat{\mathrm{ea}} /)_{3} ;$

[4] dans la structure du radical flexionnel des substantifs du type MUNTEAN, MĂCĂLEANDRU, MIREASĂ, SEARĂ, LEAFĂ, MĂSEA, MINTEAN, et des adjectifs du type VICLEAN, en se réalisant alors aussi comme l'alternance directionnelle $\boldsymbol{d V} \boldsymbol{A}_{\mathbf{8 . 4}} / \widehat{\mathrm{ea}} / \rightarrow / \mathrm{e} /$;

[5] dans la structure du radical flexionnel du verbe A ÎNVEŞTE et des adjectifs ŞUGUBĂŢ, RĂU, tout en faisant partie de l'alternance trimembre $V A_{2} / \mathrm{e} / \sim / \widehat{\mathrm{ea}} / \sim / \mathrm{o} /$;

[6] dans la structure du radical flexionnel des verbes du type A PĂREA, A MÂNEA, A VEDEA, A TĂCEA, tout en faisant partie de l'alternance trimembre $V A_{3} / \mathrm{e} / \sim / \widehat{\text { ea }} / \sim \varnothing$.

$V A_{9} \quad$ l'alternance $/ \mathrm{o} / \sim / \mathrm{oa} /$ qui se produit

[1] dans la structure du radical flexionnel des lexèmes de différentes classes grammaticales du type COVOR, UŞOR, LOTRU, RODITOR, 
MĂROI, TOT, A ÎNGROPA, A DORMI, A RĂSCOLI, A ÎNDOI, A OMORî, en se réalisant aussi comme l'alternance directionnelle $d V A_{9.1}(/ \mathbf{o} / \rightarrow / \widehat{\mathbf{o a}} /)_{1}$;

[2] dans la structure du radical flexionnel du verbe A FORFECA, en se réalisant dans ce cas aussi comme l'alternance directionnelle $\boldsymbol{d} \boldsymbol{V A}_{\mathbf{9 . 2}}$ $(/ \mathbf{o} / \rightarrow / \widehat{\mathbf{o a}} /)_{2}$;

[3] dans la structure du radical flexionnel des lexèmes de différentes classes grammaticales du type SOARE, GROAPĂ, FLOARE, FAVOARE, MOALE, A CUNOAŞTE, A SCOATE, A TOARCE, A COACE, en se réalisant alors aussi comme l'alternance directionnelle $d V A_{\mathbf{9 . 3}} / \widehat{\mathbf{o a}} / \rightarrow / \mathbf{0} /$;

[4] dans la structure du radical flexionnel des verbes du type A PURTA, A JUCA, A DESPUIA, A DUREA, A MURI, A JUPUI, A COASE, tout en faisant partie de l'alternance trimembre $V A_{1} / \mathrm{u} / \sim / \mathrm{o} / \sim / \widehat{\mathrm{oa}} /$.

I-6. les alternances bimembres non-monophonémiques réductrices : ne sont pas présentes dans le système.

I-7. les alternances bimembres monophonémiques non-réductrices :

$V A_{10}$ l'alternance $/ \mathrm{i} / \sim / \mathrm{i} /$ qui se produit dans la structure du radical flexionnel des lexèmes de différentes classes grammaticales du type LIPITOR, VIŞINĂ, MINUT, FIRESC, NIMENI, A CITI, en se réalisant aussi comme l'alternance directionnelle $\boldsymbol{d V A _ { 1 0 }} / \mathbf{i} / \rightarrow / \mathbf{i} /$.

$V A_{11}$ l'alternance $/ \mathrm{u} / \sim / \mathrm{u} /$ qui se produit dans la structure du radical flexionnel des lexèmes de différentes classes grammaticales du type CUSCRU, TRUDĂ, BUTOI, FUNEBRU, CUTARE, A DENUMI, en se réalisant aussi comme l'alternance directionnelle $\boldsymbol{d} \boldsymbol{V} \boldsymbol{A}_{11} / \mathbf{u} / \rightarrow / \mathbf{u} /$.

$V A_{12}$ l'alternance $/ \mathrm{u} / \sim / \mathrm{a} /$ qui se produit dans la structure du radical flexionnel du substantif ATU, en se réalisant aussi comme l'alternance directionnelle $d V A_{12} / \mathbf{u} / \rightarrow / \mathbf{a} /$.

$V A_{13}$ l'alternance $/ \mathbf{i} / \sim / \mathbf{i} /$ qui se produit dans la structure du radical flexionnel des lexèmes de différentes classes grammaticales du type CÂINE, CRÂŞMĂ, CÂNTEC, HÂTRU, CÂT, A VÂRî, en se réalisant aussi comme l'alternance directionnelle $d V A_{13} / \mathbf{i} / \rightarrow / \mathbf{i} /$.

$V A_{14}$ l'alternance $/ \mathbf{i} / \sim / \mathrm{a} /$ qui se produit

[1] dans la structure du radical flexionnel du substantif PÂRÂU, en se réalisant aussi comme l'alternance directionnelle $\boldsymbol{d} \boldsymbol{V} \boldsymbol{A}_{\mathbf{1 4}} / \mathbf{i} / \rightarrow / \mathbf{a} /$;

[2] dans la structure du radical flexionnel des verbes du type A RĂMÂNE et A MÂNEA, tout en faisant partie de l'alternance trimembre $V A_{4} / \mathbf{i} / \sim / \mathrm{a} /$ $\sim / \mathrm{a} /$.

$V A_{15} \quad$ l'alternance $/ \mathbf{i} / \sim / \mathrm{i} /$ qui se produit

[1a] dans la structure des suffixes participiaux (roum.: gerunziu) définitoires pour les verbes du type A OCUPA A ÎMBIA, A COMITE A SCRIE, [1b] dans la structure du radical flexionnel des substantifs du type TÂNĂR, SĂMÂNȚĂ, CUVÂNT, et des adjectifs du type SFÂNT, 
en se réalisant dans les deux cas aussi comme l'alternance directionnelle $\boldsymbol{d} \boldsymbol{V} \boldsymbol{A}_{15.1} / \mathbf{i} / \rightarrow / \mathbf{i} /$;

[2] dans la structure du radical flexionnel des verbes A VINDE et A REVINDE, en se réalisant alors aussi comme l'alternance directionnelle $\boldsymbol{d} \boldsymbol{V} \boldsymbol{A}_{15.2} / \mathbf{i} / \rightarrow / \mathbf{i} /$.

$V A_{16}$ l'alternance /a/ /a/ qui se produit dans la structure du radical flexionnel des lexèmes de différentes classes grammaticales du type ABATE, VERANDĂ, NECAZ, ZAHAROS, ALT, A ELABORA, en se réalisant aussi comme l'alternance directionnelle $\boldsymbol{d} \boldsymbol{V} \boldsymbol{A}_{16} / \mathbf{a} / \rightarrow / \mathbf{a} /$.

$V A_{17}$ l'alternance $/ \mathrm{a} / \sim / \mathrm{e} /$ qui se produit

[1] dans la structure du radical flexionnel des substantifs du type LICEAN, PUIANDRU, MASĂ, VARĂ, ŞA, LIGHEAN, ŞARPE, AMBREIARE, et des adjectifs du type COTIDIAN, en se réalisant aussi comme l'alternance directionnelle $\boldsymbol{d} V \boldsymbol{A}_{17.1}(/ \mathbf{a} / \rightarrow / \mathrm{e} /)_{1}$;

[2] dans la structure du suffixe de parfait définitoire pour les verbes du type A DEOCHEA, A ÎNCUIA, A ÎNTÂRZIA, A VEGHEA, A ÎNTÂRZIA, A STUDIA, en se réalisant dans ce cas aussi comme l'alternance directionnelle $\boldsymbol{d} \boldsymbol{V} \boldsymbol{A}_{\mathbf{1 7 . 2}}(/ \mathrm{a} / \rightarrow / \mathrm{e} /)_{2}$;

[3] dans la structure du radical flexionnel des verbes du type A AIEPTA, A ÎNTEMEIA, A STUDIA, A PIERDE, A FIERBE, A IEŞI, A PUSTII, et des adjectifs du type MĂIESTRU, en se réalisant dans ce cas aussi comme l'alternance directionnelle $\boldsymbol{d} \boldsymbol{V} \boldsymbol{A}_{\mathbf{1 7 . 3}}(/ \mathrm{e} / \rightarrow / \mathbf{a} /)_{1}$;

[4] dans la structure du radical flexionnel du verbe A FERMECA, en se réalisant dans ce cas aussi comme l'alternance directionnelle $\boldsymbol{d} \boldsymbol{V} \boldsymbol{A}_{\mathbf{1 7 . 4}}$ $(/ \mathbf{e} / \rightarrow / \mathbf{a} /)_{2}$;

[5] dans la structure du radical flexionnel des verbes du type A DEŞERTA, A ŞEDEA, et des substantifs du type FIER, en se réalisant alors aussi comme l'alternance directionnelle $\boldsymbol{d} \boldsymbol{V} \boldsymbol{A}_{\mathbf{1 7 . 5}}(/ \mathrm{e} / \rightarrow / \mathbf{a} /)_{3}$;

[6] dans la structure du radical flexionnel des verbes du type A ÎNVĂŢA, A ÎMBĂTA, A APĂSA, A VEDEA, tout en faisant partie de l'alternance trimembre $V A_{5} / \mathrm{a} / \sim / \mathrm{e} / \sim / \mathrm{a} /$;

[7] dans la structure du radical flexionnel des verbes du type A DEOCHEA, A DESFOIA, A ÎNTÂRZIA, A VEGHEA, A ÎNTEMEIA, A STUDIA, tout en faisant partie de l'alternance trimembre $V A_{6} / \mathrm{a} / \sim / \mathrm{e} / \sim \varnothing$.

$V A_{18}$ l'alternance /a/ /a/ qui se produit

[1] dans la structure du radical flexionnel des substantifs du type ALAMĂ, GARĂ, MARE, CARNE, LAPTE, MUCAR, CORABIE, des adjectifs du type LAI, CĂLARE, et des pronoms du type CARE, CUTARE, en se réalisant aussi comme l'alternance directionnelle $\boldsymbol{d} \boldsymbol{V} \boldsymbol{A}_{\mathbf{1 8 . 1}}(/ \mathbf{a} / \rightarrow / \mathbf{o} /)_{1}$;

[2] dans la structure du radical flexionnel des verbes du type A BATE, A RADE, A SPARGE, en se réalisant dans ce cas aussi comme l'alternance directionnelle $d V A_{18.2}(/ \mathbf{a} / \rightarrow / \partial /)_{2}$; 
[3] dans la structure du suffixe de parfait définitoire pour les verbes du type A OCUPA, A ARUNCA, A UMBLA, A CONTINUA, A LUCRA, A PARCA, A PIUA, A EFECTUA, en se réalisant dans ce cas aussi comme l'alternance directionnelle $d V A_{18.3}(/ \mathbf{a} / \rightarrow / \mathrm{o} /)_{3}$;

[4] dans la structure du radical flexionnel des verbes du type A ÎNĂLŢA, A PĂREA, A SĂRI, A TĂBĂRî, des pronoms du type TĂU, et du substantif PÂRĂU, en se réalisant dans ce cas aussi comme l'alternance directionnelle $d V A_{18.4}(/ \mathbf{a} / \rightarrow / \mathbf{a} /)_{1}$;

[5] dans la structure du radical flexionnel des verbes du type A AGĂȚA, A ARĂTA, A ADĂSTA, en se réalisant dans ce cas aussi comme l'alternance directionnelle $\boldsymbol{d} \boldsymbol{V} \boldsymbol{A}_{18.5}(/ \mathrm{a} / \rightarrow / \mathrm{a} /)_{2}$;

[6] dans la structure du radical flexionnel des verbes du type A HOTĂRî, en se réalisant alors aussi comme l'alternance directionnelle $d V A_{18.6}(/ / /$ $\rightarrow / \mathbf{a} /)_{3}$;

[7] dans la structure du radical flexionnel des verbes du type A RĂMÂNE et A MÂNEA, tout en faisant partie de l'alternance trimembre $V A_{4} / \mathbf{i} / \sim / \mathrm{a} /$ $\sim / \mathrm{a} /$

[8] dans la structure du radical flexionnel des verbes du type A ÎNVĂȚA, A ÎMBĂTA, A APĂSA, A VEDEA, tout en faisant partie de l'alternance trimembre $V A_{5} / \mathrm{a} / \sim / \mathrm{e} / \sim / \mathrm{a} /$;

[9] dans la structure du radical flexionnel des verbes du type A OCUPA, A ARUNCA, A UMBLA, A CONTINUA, A LUCRA, A PARCA, A PIUA, A EFECTUA, tout en faisant partie de l'alternance trimembre $V A_{7} / \mathrm{a} / \sim$ $/ \partial / \sim \varnothing$.

$V A_{19}$ l'alternance /e/ /e/ qui se produit dans la structure du radical flexionnel des lexèmes de différentes classes grammaticales du type PERETE, VERANDĂ, NECAZ, FERVENT, A DENUMI, en se réalisant aussi comme l'alternance directionnelle $\boldsymbol{d V A _ { 1 9 }} / \mathrm{e} / \rightarrow / \mathrm{e} /$.

$V A_{20}$ l'alternance /e/ $\sim /$ i/ qui se produit dans la structure du radical flexionnel des verbes du type A PREZENTA, A VENI, en se réalisant aussi comme l'alternance directionnelle $\boldsymbol{d V A _ { 2 0 }} / \mathbf{e} / \rightarrow / \mathrm{i} /$.

$V A_{21} \quad$ l'alternance $/ \mathrm{o} / \sim / \mathrm{o} /$ qui se produit dans la structure du radical flexionnel des lexèmes de différentes classes grammaticales du type LOTRU, COLIBĂ, BORCAN, DOMESTIC, NOI, A OCUPA, en se réalisant aussi comme l'alternance directionnelle $d V A_{21} / \mathbf{o} / \rightarrow / \mathbf{o} /$.

$V A_{22}$ l'alternance $/ \mathrm{o} / \sim / \mathrm{a} /$ qui se produit dans la structure du radical flexionnel du substantif CARO, en se réalisant aussi comme l'alternance directionnelle $\boldsymbol{d V} \boldsymbol{A}_{22} / \mathbf{0} / \rightarrow / \mathbf{a} /$.

$V A_{23}$ l'alternance $/ \mathrm{\partial} / \sim / \mathrm{a} /$ qui se produit dans la structure du radical flexionnel des lexèmes de différentes classes grammaticales du type CĂLUGĂR, CĂMAŞĂ, GRĂTAR, NĂTÂNG, A PĂCĂLI, en se réalisant aussi comme l'alternance directionnelle $\boldsymbol{d V} \boldsymbol{A}_{\mathbf{2 3}} / \mathbf{a} / \rightarrow / \mathrm{a} /$. 
$V A_{24}$ l'alternance /a/ /e/ qui se produit

[1] dans la structure du radical flexionnel des lexèmes de différentes classes grammaticales du type PĂR, CUMĂTRU, SĂMÂNŢĂ, BĂȚ, CĂPĂSTRU, RĂU, TÂNĂR, A CUMPĂRA, A TĂBĂRî, en se réalisant aussi comme l'alternance directionnelle $\boldsymbol{d V} \boldsymbol{A}_{\mathbf{2 4 . 1}}(\mathrm{/} / \mathrm{a} \rightarrow / \mathrm{e} /)_{1}$;

[2] dans la structure du radical flexionnel du verbe A ZGREPȚĂNA, en se réalisant dans ce cas aussi comme l'alternance directionnelle $\boldsymbol{d} \boldsymbol{V} \boldsymbol{A}_{\mathbf{2 4 . 2}}$ $(/ \mathrm{a} / \rightarrow / \mathrm{e} /)_{2}$

[3] dans la structure des désinences de présent définitoires pour les verbes du type A OCUPA $\sim$ A ÎMBIA, A COMITE $\sim$ A SCRIE, en se réalisant dans ce cas aussi comme l'alternance directionnelle $d V A_{24.3}(/ \mathbf{a} / \rightarrow / \mathbf{e} /)_{3}$;

[4] dans la structure du radical flexionnel des verbes du type A ACOPERI, en se réalisant alors aussi comme l'alternance directionnelle $\boldsymbol{d} \boldsymbol{V} \boldsymbol{A}_{\mathbf{2 4 . 4}}$ $/ \mathrm{e} / \rightarrow / \mathrm{o} /$

[5] dans la structure du radical flexionnel du verbe A ÎNVEŞTE et des adjectifs ŞUGUBĂŢ, RĂU, tout en faisant partie de l'alternance trimembre $V A_{2} / \mathrm{e} / \sim / \overline{\mathrm{ea}} / \sim / \mathrm{a} /$;

[6] dans la structure du radical flexionnel des verbes du type A ÎNVĂŢA, A ÎMBĂTA, A APĂSA, A VEDEA, tout en faisant partie de l'alternance trimembre $V A_{5} / \mathrm{a} / \sim / \mathrm{e} / \sim / \mathrm{a} /$.

I-8. les alternances bimembres monophonémiques réductrices :

$V A_{25}$ l'alternance $/ \mathrm{i} / \sim \varnothing$ qui se produit dans la structure du radical flexionnel des verbes du type A ADORMI, A OFERI, A BIRUI, A IUBI, A LOCUI, en se réalisant aussi comme l'alternance directionnelle $d V A_{25} / \mathbf{i} / \rightarrow \boldsymbol{\emptyset}$.

$V A_{26}$ l'alternance $/ \mathrm{u} / \sim \emptyset$ qui se produit dans la structure des désinences [a] de singulier définitoires pour les substantifs du type COPIL, CRAI, PAHAR, TRAMVAI, PORTCHEI, DULAP, TEMEI, [b] de présent 1sg définitoires pour les verbes du type A UMBLA $\sim$ A OCUPA, A UMPLE $\sim$ A BATE, A ZVÂRLI A ADORMI, en se réalisant aussi comme l'alternance directionnelle $\boldsymbol{d} \boldsymbol{V} \boldsymbol{A}_{\mathbf{2 6}}$ $/ \mathbf{u} / \sim \varnothing$.

$V A_{27} \quad$ l'alternance $/ \mathbf{i} / \sim \varnothing$ qui se produit

[1] dans la structure du radical flexionnel des verbes du type A VÂRÎ, A HOTĂRî, en se réalisant aussi comme l'alternance directionnelle $d V A_{27.1}(/ \mathbf{i} / \rightarrow \emptyset)_{1}$

[2] dans la structure des pronoms du type ÎMI, ÎȚI, ÎșI, en se réalisant alors aussi comme l'alternance directionnelle $d V A_{27.2}(\mathbf{i} / / \rightarrow \emptyset)_{2}$.

$V A_{28}$ l'alternance $/ \mathrm{e} / \sim \varnothing$ qui se produit

[1] dans la structure du radical flexionnel des verbes du type A BATE, A COMITE, A RUPE, en se réalisant aussi comme l'alternance directionnelle $d V A_{28.1}(/ \mathrm{e} / \rightarrow \emptyset)_{1}$; 
[2a] dans la structure des suffixes de parfait définitoires pour les verbes du type A ADUCE, A COMITE, A SCRIE; [2b] dans la structure du pronom SE, en se réalisant dans ces deux derniers cas aussi comme l'alternance directionnelle $\boldsymbol{d} \boldsymbol{V} \boldsymbol{A}_{\mathbf{2 8 . 2}}(/ \mathrm{e} / \rightarrow \boldsymbol{\emptyset})_{2}$.

$V A_{29}$ l'alternance $/ \mathrm{o} / \sim \varnothing$ qui se produit dans la structure du radical flexionnel des substantifs SOMBRERO et FLAMINGO, en se réalisant aussi comme l'alternance directionnelle $d V A_{29} / \mathbf{o} / \rightarrow \emptyset$.

$V A_{30}$ l'alternance $/ \mathrm{a} / \sim \emptyset$ qui se produit

[1a] dans la structure des désinences de singulier définitoires pour les substantifs du type FATĂ, LIPSĂ, PĂSĂRICĂ, INIMĂ, TREABĂ, [1b] dans la structure des pronoms MĂ, VĂ, en se réalisant dans ces deux cas aussi comme l'alternance directionnelle $d V A_{30.1}(/ / / \rightarrow \emptyset)_{1}$;

[2] dans la structure des pronoms CELĂLALT et CESTĂLALT, en se réalisant alors aussi comme l'alternance directionnelle $d V A_{30.2}(/ \mathbf{a} / \rightarrow \emptyset)_{2}$.

\section{ALTERNANCES NON-CONSONANTIQUES TRANSITOIRES (QUI SONT TOUJOURS BIMEMBRES MONOPHONEMIQUES NON-REDUCTRICES) :}

$T A_{1} \quad$ l'alternance $/ \mathrm{i} / \sim / \mathrm{j} /$ qui se produit

[1a] dans la structure des suffixes de pluriel définitoires pour les substantifs du type ŢĂRAN, COPIL, NUNŢIU, EROU, FRATE, LIPSĂ, INIMĂ, TREABĂ, VULPE, PIELE, ALEE, BESTIE, VREME, SCUMPETE, ŞA, FLUVIU, CASIU, DULAP, TEMEI, [1b] dans la structure des désinences de présent 1 sg définitoires pour les verbes du type A UMBLA A OCUPA, A UMPLE $\sim$ A RUPE, A ZVÂRLI $\sim$ A DORMI, [1c] dans la structure des pronoms du type ÎMI, ÎȚI, ÎșI, ÎI, en se réalisant dans ces trois cas aussi comme l'alternance directionnelle $\boldsymbol{d T} \boldsymbol{A}_{\mathbf{1 . 1}} / \mathbf{i} / \rightarrow / \mathbf{j} /$;

[2] dans la structure du radical flexionnel des substantifs du type NUNŢIU, FLUVIU, et des adjectifs du type PROPRIU, en se réalisant alors aussi comme l'alternance directionnelle $\boldsymbol{d T} \boldsymbol{A}_{1.2} / \mathbf{j} / \rightarrow / \mathbf{i} /$.

$T A_{2}$ l'alternance $/ \mathrm{u} / \sim / \mathrm{w} /$ qui se produit

[1a] dans la structure des désinences de singulier définitoires pour les substantifs (et des adjectifs) du type EROU, LICEU, CURCUBEU, CASIU, STILOU, [1b] dans la structure des désinences de présent définitoires pour les verbes du type A UMBLA $\sim$ A CONTINUA, A UMPLE $\sim$ A SCRIE, A ZVÂRLI $\sim$ A ŞTI, en se réalisant dans les deux cas aussi comme

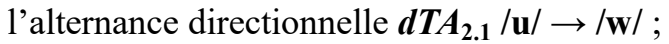

[2] dans la structure du substantif oU, en se réalisant alors aussi comme l'alternance directionnelle $\boldsymbol{d T A} \boldsymbol{A}_{2.2} / \mathbf{w} / \rightarrow / \mathbf{u} /$. 
$T A_{3}$ l'alternance /o/ / w/ qui se produit dans la structure du radical flexionnel du substantif CACAO, en se réalisant aussi comme l'alternance directionnelle $\boldsymbol{d T} \boldsymbol{A}_{3} / \mathbf{o} / \rightarrow / \mathrm{w} /$.

$T A_{4} \quad$ l'alternance $/ \mathrm{j} / \sim / \mathrm{j} /$ qui se produit dans la structure du radical flexionnel des lexèmes de différentes classes grammaticales du type IEPURE, IESLE, IAURT, IERBOS, EL, A MĂIESTRI, en se réalisant aussi comme l'alternance directionnelle $\boldsymbol{d T} \boldsymbol{A}_{\mathbf{4}} / \mathbf{j} / \rightarrow / \mathbf{j} /$.

$T A_{5}$ l'alternance $/ \mathrm{w} / \sim / \mathrm{w} /$ qui se produit dans la structure du radical flexionnel des lexèmes de différentes classes grammaticales du type QUASAR, ACUARELĂ, QUARK, CUATROCENTIST, A PIUA, en se réalisant aussi comme l'alternance directionnelle $\boldsymbol{d T \boldsymbol { A } _ { 5 }} / \mathbf{w} / \rightarrow / \mathbf{w} /$.

\section{ALTERNANCES CONSONANTIQUES}

III-1. les alternances non-bimembres non-trimembres non-quadrimembres : ne sont pas présentes dans le système.

III-2. les alternances non-bimembres non-trimembres quadrimembres non-monophonémiques : ne sont pas présentes dans le système.

III-3. les alternances non-bimembres non-trimembres quadrimembres monophonémiques non-réductrices : ne sont pas présentes dans le système.

III-4. les alternances non-bimembres non-trimembres quadrimembres monophonémiques réductrices :

$C A_{1} \quad$ l'alternance $/ \mathrm{d} / \sim / \mathrm{z} / \sim / \mathrm{g} / \sim \varnothing$ qui se produit dans la structure du radical flexionnel des verbes A UCIDE, A SINUCIDE, en se réalisant aussi comme

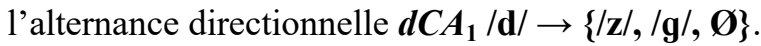

III-5. les alternances non-bimembres trimembres non-monophonémiques : ne sont pas présentes dans le système.

III-6. les alternances non-bimembres trimembres monophonémiques non-réductrices :

$C A_{2}$ l'alternance $/ \mathrm{g} / \sim / \mathrm{d} / / \sim / \mathrm{p} /$ qui se produit dans la structure du radical flexionnel des verbes A SUGE, A FRIGE, A ÎNFIGE, en se réalisant aussi comme l'alternance directionnelle $\boldsymbol{d} \boldsymbol{C A}_{\mathbf{2}} / \mathbf{d} \mathbf{z} / \rightarrow\{/ \mathbf{g} /, / \mathbf{p} /\}$.

$C A_{3}$ l'alternance $/ \mathrm{k} / \sim / \mathrm{g} / \sim / \mathrm{p} /$ qui se produit dans la structure du radical flexionnel des verbes A COACE, A RĂSCOACE, A RECOACE, en se réalisant aussi comme l'alternance directionnelle $\boldsymbol{d} \boldsymbol{C A}_{\mathbf{3}} / \mathbf{t} / \rightarrow\{/ \mathbf{k} /, / \mathbf{p} /\}$.

III-7. les alternances non-bimembres trimembres monophonémiques réductrices :

$C A_{4}$ l'alternance $/ \mathrm{g} / \sim / \mathrm{d}_{3} / \sim \varnothing$ qui se produit dans la structure du radical flexionnel des verbes du type A TRAGE, A STINGE, A ALEGE, A FRÂNGE, A SPARGE, en se réalisant aussi comme l'alternance directionnelle $\boldsymbol{d} \boldsymbol{C A}_{4}$ $/ \mathbf{d} \mathbf{3} / \rightarrow\{/ \mathbf{g} /, \boldsymbol{\varnothing}\}$. 
$C A_{5}$ l'alternance $/ \mathrm{k} / \sim / \mathfrak{g} / \sim \varnothing$ qui se produit dans la structure du radical flexionnel des verbes du type A TOARCE, A FACE, A ADUCE, en se réalisant aussi comme l'alternance directionnelle $\boldsymbol{d} \boldsymbol{C A}_{\mathbf{5}} / \mathbf{t} / \rightarrow\{/ \mathbf{k} /, \boldsymbol{O}\}$.

$C A_{6}$ l'alternance $/ \mathrm{d} / \sim / \mathrm{z} / \sim \varnothing$ qui se produit dans la structure du radical flexionnel des verbes du type A PRINDE, A RADE, A PURCEDE, A ROADE, en se réalisant aussi comme l'alternance directionnelle $\boldsymbol{d C \boldsymbol { A } _ { 6 }} / \mathbf{d} / \rightarrow\{/ \mathbf{z} /, \boldsymbol{\emptyset}\}$.

$\mathrm{CA}_{7}$ l'alternance $/ \mathrm{t} / \sim / \mathrm{ts} / \sim \varnothing$ qui se produit dans la structure du radical flexionnel des verbes du type A COMITE, A SUMETE, A SCOATE, en se

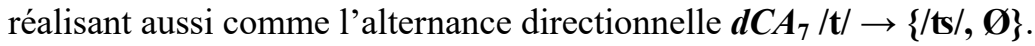

III-8. les alternances bimembres non-monophonémiques non-réductrices :

$C A_{8} \quad$ l'alternance $/ \widehat{\mathrm{st}} / \sim / \widehat{\mathrm{ft}} / \mathrm{qui}$ se produit dans la structure du radical flexionnel des lexèmes de différentes classes grammaticales du type ARBUST, ASTRU, NĂPASTĂ, VESTE, TRIST, ACEST, CESTĂLALT, A EXISTA, A ADĂSTA, en se

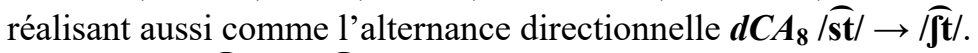

$C A_{9}$ l'alternance $/ \widehat{\mathrm{sk}} / \sim \widehat{\mathrm{st}} /$ qui se produit dans la structure du radical flexionnel des substantifs et des adjectifs du type ETRUSC, FRESCĂ,

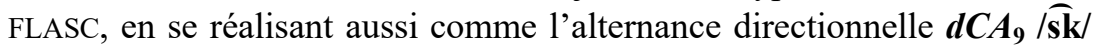

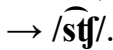

$C A_{10}$ 1'alternance $/ \widehat{\mathrm{sk}} / \sim / \widehat{\mathrm{ft}} /$ qui se produit

[1] dans la structure du radical flexionnel des lexèmes de différentes classes grammaticales du type MOSC, MUSCĂ, PLOSCĂ, BRUSC, LIVRESC, A CONFISCA, A CĂSCA, A IUBI, A LOCUI, A HOTĂRÎ, A TREBUI, en se réalisant aussi comme l'alternance directionnelle $\boldsymbol{d C A _ { 1 0 . 1 } / \mathbf { s k } /} \rightarrow / \widehat{\int t} /$;

[2] dans la structure du radical flexionnel des verbes du type A NAŞTE, A CREŞTE, A CUNOAŞTE, A ÎNVEŞTE, en se réalisant alors aussi comme l'alternance directionnelle $\boldsymbol{d C \boldsymbol { A } _ { 1 0 . 2 } / \widehat { \int } \mathbf { t } /} \rightarrow / \widehat{\mathbf{s k}} /$.

$C A_{11}$ l'alternance $/ \widehat{\int k} / \sim \sqrt{\int \mathrm{g}} /$ qui se produit dans la structure du radical flexionnel du substantif DEŞCĂ, en se réalisant aussi comme l'alternance directionnelle $\boldsymbol{d} \boldsymbol{C} \boldsymbol{A}_{\mathbf{1 1}} / \widehat{\mathbf{J k}} / \rightarrow / \widehat{\int} \mathbf{t} /$.

$C A_{12}$ l'alternance $/ \widehat{\int k} / \sim / \sqrt{\mathrm{t}} /$ qui se produit dans la structure du radical flexionnel des substantifs du type GĂLUŞCĂ, CLOŞCĂ, en se réalisant aussi comme l'alternance directionnelle $\boldsymbol{d} \boldsymbol{C A}_{\mathbf{1 2}} / \widehat{\mathbf{J k}} / \rightarrow / \widehat{\mathbf{J}} /$.

III-9. les alternances bimembres non-monophonémiques réductrices : ne sont pas présentes dans le système.

III-10. les alternances bimembres monophonémiques non-réductrices :

$C A_{13}$ l'alternance $/ \mathrm{m} / \sim / \mathrm{m} /$ qui se produit dans la structure du radical flexionnel des lexèmes de différentes classes grammaticales du type $\mathrm{OM}$, MASĂ, ANSAMBLU, MÂNDRU, NIMENI, A MIROSI, en se réalisant aussi comme l'alternance directionnelle $\boldsymbol{d C A _ { 1 3 }} / \mathbf{m} / \rightarrow / \mathbf{m} /$.

$C A_{14}$ l'alternance $/ \mathrm{n} / \sim / \mathrm{n} /$ qui se produit dans la structure du radical flexionnel des lexèmes de différentes classes grammaticales du type CĂRBUNE, 
DONIŢĂ, TANC, LUNAR, NOI, A FONFĂI, en se réalisant aussi comme l'alternance directionnelle $\boldsymbol{d} \boldsymbol{C} \boldsymbol{A}_{\mathbf{1 4}} / \mathbf{n} / \rightarrow / \mathbf{n} /$.

$C A_{15}$ l'alternance $/ 1 / \sim / 1 /$ qui se produit dans la structure du radical flexionnel des lexèmes de différentes classes grammaticales du type CHELNER, ECLIPSĂ, SĂLAŞ, MELODIC, ALT, A ELABORA, en se réalisant aussi comme l'alternance directionnelle $\boldsymbol{d} \boldsymbol{C A}_{15} / \mathbf{l} / \rightarrow / \mathbf{l} /$.

$C A_{16}$ l'alternance $/ \mathrm{r} / \sim / \mathrm{r} /$ qui se produit dans la structure du radical flexionnel des lexèmes de différentes classes grammaticales du type CERB, DRUPĂ, SĂRUT, MEREU, CARE, A IRITA, en se réalisant aussi comme l'alternance directionnelle $\boldsymbol{d} \boldsymbol{C} \boldsymbol{A}_{16} / \mathbf{r} / \rightarrow / \mathbf{r} /$.

$C A_{17}$ l'alternance $/ \mathrm{v} / \sim / \mathrm{v} /$ qui se produit dans la structure du radical flexionnel des lexèmes de différentes classes grammaticales du type COVALI, DUMBRAVĂ, SCLAVAJ, MOSCOVIT, VREUN, A IZVODI, en se réalisant aussi comme l'alternance directionnelle $\boldsymbol{d C \boldsymbol { A } _ { 1 7 }} / \mathrm{v} / \rightarrow / \mathrm{v} /$.

$C A_{18}$ l'alternance $/ \mathrm{f} / \sim / \mathrm{f} /$ qui se produit dans la structure du radical flexionnel des lexèmes de différentes classes grammaticales du type ELEFANT, FATĂ, CALORIFER, NECROFIL, FIECARE, A ÎNFOCA, en se réalisant aussi comme l'alternance directionnelle $\boldsymbol{d} \boldsymbol{C} \boldsymbol{A}_{\mathbf{1 8}} / \mathbf{f} / \rightarrow / \mathbf{f} /$.

$C A_{19}$ l'alternance $/ \mathrm{z} / \sim / \mathrm{z} /$ qui se produit dans la structure du radical flexionnel des lexèmes de différentes classes grammaticales du type FAZAN, EREZIE, CAZ, DIVIZAT, A ÎNFRUNZI, en se réalisant aussi comme l'alternance directionnelle $\boldsymbol{d} \boldsymbol{C} \boldsymbol{A}_{19} / \mathbf{z} / \rightarrow / \mathbf{z} /$.

$C A_{20}$ l'alternance $/ z / \sim / 3 /$ qui se produit dans la structure du radical flexionnel des substantifs du type OBRAZ, en se réalisant aussi comme l'alternance directionnelle $\boldsymbol{d} \boldsymbol{C A}_{\mathbf{2 0}} / \mathbf{z} / \rightarrow / 3 /$.

$C A_{21}$ l'alternance $/ \mathrm{s} / \sim / \mathrm{s} /$ qui se produit dans la structure du radical flexionnel des lexèmes de différentes classes grammaticales du type GÂSCAN, CASĂ, CEAS, ESTETIC, SIE, A ÎNSCRIE, en se réalisant aussi comme l'alternance directionnelle $\boldsymbol{d} \boldsymbol{C} \boldsymbol{A}_{21} / \mathbf{s} / \rightarrow / \mathbf{s} /$.

$C A_{22}$ l'alternance $/ \mathrm{s} / \sim / \mathrm{J} /$ qui se produit

[1] dans la structure du radical flexionnel des substantifs du type URS, SFINX, et des adjectifs du type GRAS, FIX, en se réalisant aussi comme l'alternance directionnelle $\boldsymbol{d} \boldsymbol{C A}_{\mathbf{2 2 . 1}}(/ \mathbf{s} / \rightarrow / \mathrm{J} /)_{1}$;

[2] dans la structure du radical flexionnel des verbes du type A LĂSA, A APĂSA, A ŢESE, A COASE, A MIROSI, en se réalisant dans ce cas aussi comme l'alternance directionnelle $\boldsymbol{d} \boldsymbol{C \boldsymbol { A } _ { 2 2 . 2 }}(/ \mathrm{s} / \rightarrow / \mathbf{J} /)_{2}$;

[3] dans la structure du radical flexionnel des verbes du type A IEŞI, en se réalisant alors aussi comme l'alternance directionnelle $\boldsymbol{d} \boldsymbol{C A}_{\mathbf{2 2 . 3}}$ $/ \mathbf{f} / \rightarrow / \mathbf{s} /$.

$C A_{23}$ l'alternance $/ 3 / \sim / 3 /$ qui se produit dans la structure du radical flexionnel des lexèmes de différentes classes grammaticales du type JUPÂN, 
ETAJERĂ, COJOC, JUST, A JUCA, en se réalisant aussi comme l'alternance directionnelle $\boldsymbol{d} \boldsymbol{C A}_{23} / 3 / \rightarrow / 3 /$.

$C A_{24}$ l'alternance $/ \delta / \sim / \delta /$ qui se produit dans la structure du radical flexionnel des lexèmes de différentes classes grammaticales du type MĂNUŞAR, FIŞĂ, COŞMAR, OBIŞNUIT, A LEŞINA, en se réalisant aussi comme l'alternance

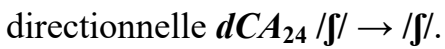

$C A_{25}$ l'alternance $/ \mathrm{x} / \sim / \mathrm{x} /$ qui se produit dans la structure du radical flexionnel des lexèmes de différentes classes grammaticales du type HOȚ, HÂRTIE, HOHOT, HAZLIU, A LIHNI, en se réalisant aussi comme l'alternance directionnelle $\boldsymbol{d} \boldsymbol{C A}_{25} / \mathbf{x} / \rightarrow / \mathbf{x} /$.

$C A_{26}$ l'alternance $/ \mathrm{x} / \sim / \mathrm{g} /$ qui se produit dans la structure du radical flexionnel $\mathrm{du}$ substantif LEAH, en se réalisant aussi comme l'alternance directionnelle $\boldsymbol{d C A} \boldsymbol{A}_{26} / \mathbf{x} / \rightarrow / \mathrm{J} /$.

$C A_{27}$ l'alternance /ts/ $\sim / \mathrm{s} /$ qui se produit dans la structure du radical flexionnel des lexèmes de différentes classes grammaticales du type ȚÂNŢAR, TARĂ, ŢĂRM, ȚĂRĂNESC, ȚIE, A ȚESE, en se réalisant aussi comme l'alternance

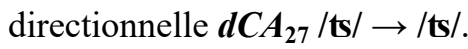

$C A_{28}$ l'alternance $/ \mathrm{d} / \sim / \mathrm{d} /$ qui se produit dans la structure du radical flexionnel des lexèmes de différentes classes grammaticales du type GENUNCHI, GIRAFĂ, GEAM, GINGAŞ, A GEME, en se réalisant aussi comme

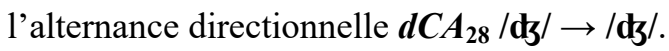

$C A_{29}$ l'alternance $/ \mathfrak{g} / \sim / \mathfrak{t} /$ qui se produit dans la structure du radical flexionnel des lexèmes de différentes classes grammaticales du type CIOCAN, CETATE, CERC, CINSTIT, ACEST, A CITI, en se réalisant aussi comme l'alternance directionnelle $\boldsymbol{d} \boldsymbol{C A}_{\mathbf{2 9}} / \mathbf{t g} / \rightarrow / \mathbf{g} /$.

$C A_{30}$ l'alternance $/ \mathrm{b} / \sim / \mathrm{b} /$ qui se produit dans la structure du radical flexionnel des lexèmes de différentes classes grammaticales du type, BESTIE, BLOC, BRUSC, A BOTEZA, en se réalisant aussi comme l'alternance directionnelle $d C A_{30} / \mathbf{b} / \rightarrow / \mathbf{b} /$.

$C A_{31}$ l'alternance $/ \mathrm{p} / \sim / \mathrm{p} /$ qui se produit dans la structure du radical flexionnel des lexèmes de différentes classes grammaticales du type POM, POMPĂ, PROSOP, POPULAR, A PUPA, en se réalisant aussi comme l'alternance directionnelle $\boldsymbol{d} \boldsymbol{C} \boldsymbol{A}_{31} / \mathbf{p} / \rightarrow / \mathbf{p} /$.

$C A_{32}$ l'alternance $/ \mathrm{d} / \sim / \mathrm{d} /$ qui se produit dans la structure du radical flexionnel des lexèmes de différentes classes grammaticales du type DESPOT, DATĂ, DULAP, MÂNDRU, A DANSA, en se réalisant aussi comme l'alternance directionnelle $\boldsymbol{d C} \boldsymbol{A}_{32} / \mathbf{d} / \rightarrow / \mathbf{d} /$.

$C A_{33}$ l'alternance $/ \mathrm{d} / \sim / \mathrm{z} /$ qui se produit

[1] dans la structure du radical flexionnel des lexèmes de différentes classes grammaticales du type BRAD, EREDE, OGLINDĂ, LESPEDE, 
SURD, VERDE, A COMANDA, A APLAUDA, A PRĂDA, en se réalisant aussi comme l'alternance directionnelle $\boldsymbol{d} \boldsymbol{C A}_{33.1}(/ \mathbf{d} / \rightarrow / \mathbf{z} /)_{1}$;

[2] dans la structure du radical flexionnel des verbes du type A PIERDE, A VINDE, en se réalisant dans ce cas aussi comme l'alternance directionnelle $\boldsymbol{d} \boldsymbol{C A}_{33.2}(/ \mathbf{d} / \rightarrow / \mathbf{z} /)_{2}$;

[3] dans la structure du radical flexionnel des verbes du type A ŞEDEA, A CĂDEA, A CREDE, A VEDEA, en se réalisant dans ce cas aussi comme l'alternance directionnelle $\boldsymbol{d} \boldsymbol{C A}_{\mathbf{3 3 . 3}}(/ \mathbf{d} / \rightarrow / \mathbf{z} /)_{3}$;

[4] dans la structure du radical flexionnel des verbes du type A AUZI, A REPEZI, A SLOBOZI, en se réalisant alors aussi comme l'alternance directionnelle $\boldsymbol{d} \boldsymbol{C A}_{\mathbf{3 3 . 4}} / \mathbf{z} / \rightarrow / \mathbf{d} /$;

[5] dans la structure du radical flexionnel des verbes A UCIDE, A SINUCIDE, tout en faisant partie de l'alternance quadrimembre $C A_{1} / \mathrm{d} / \sim / \mathrm{z} / \sim$ $/ \mathrm{g} / \sim \varnothing$.

$\mathrm{CA}_{34}$ l'alternance $/ \mathrm{d} / \sim / 3 /$ qui se produit dans la structure du radical flexionnel du substantif TRÂND, en se réalisant aussi comme l'alternance directionnelle $\boldsymbol{d} \boldsymbol{C A}_{\mathbf{3 4}} / \mathrm{d} / \rightarrow / \mathrm{z} /$.

$C A_{35}$ l'alternance $/ \mathrm{t} / \sim / \mathrm{t} /$ qui se produit dans la structure du radical flexionnel des lexèmes de différentes classes grammaticales du type TURC, TROMPĂ, PATEU, COTIDIAN, CUTARE, A PROTEJA, en se réalisant aussi comme l'alternance directionnelle $\boldsymbol{d} \boldsymbol{C} \boldsymbol{A}_{35} / \mathbf{t} / \rightarrow / \mathbf{t} /$.

$C A_{36}$ l'alternance $/ \mathrm{t} / \sim / \mathrm{ts} /$ qui se produit

[1] dans la structure du radical flexionnel des substantifs du type STUDENT, FRATE, TATĂ, NUNTĂ, CURTE, des adjectifs du type ÎNALT, IUTE, et des pronoms du type CÂT, ATÂT, MULT, TOT, ALT, en se réalisant aussi comme l'alternance directionnelle $\boldsymbol{d} \boldsymbol{C A}_{\mathbf{3 6 . 1}}(/ \mathbf{t} / \rightarrow / \mathbf{t s} /)_{1}$;

[2] dans la structure du radical flexionnel des verbes du type A AJUTA, A PUTEA, A BATE, A ÎNVÂRTI, en se réalisant dans ce cas aussi comme l'alternance directionnelle $\boldsymbol{d} \boldsymbol{C A}_{\mathbf{3 6 . 2}}(/ \mathbf{t} / \rightarrow / \mathbf{t} /)_{\mathbf{2}}$;

[3] dans la structure du radical flexionnel des verbes du type A SIMŢI, A ÎMPĂRŢI, en se réalisant alors aussi comme l'alternance directionnelle $\boldsymbol{d} \boldsymbol{C A}_{\mathbf{3 6 . 3}} / \mathbf{t s} / \rightarrow / \mathbf{t} /$;

[4] dans la structure du radical flexionnel des verbes du type A COMITE, A SUMETE, A SCOATE, tout en faisant partie de l'alternance trimembre $C A_{7} / \mathrm{t} / \sim / \mathrm{ts} / \sim \varnothing$.

$C A_{37}$ l'alternance $/ \mathrm{J} / \sim / \mathrm{f} /$ qui se produit dans la structure du radical flexionnel des lexèmes de différentes classes grammaticales du type BURGHEZ, BAGHETĂ, GHID, GHEŢOS, A STINGHERI, en se réalisant aussi comme l'alternance directionnelle $\boldsymbol{d} \boldsymbol{C A}_{\mathbf{3 7}} / \mathbf{J} / \rightarrow / \mathbf{J} /$.

$C A_{38}$ l'alternance $/ \mathrm{c} / \sim / \mathrm{c} /$ qui se produit dans la structure du radical flexionnel des lexèmes de différentes classes grammaticales du type CHIRURG, 
BRICHETĂ, PACHET, ÎNCHEIAT, A TACHINA, en se réalisant aussi comme l'alternance directionnelle $\boldsymbol{d} \boldsymbol{C A}_{38} / \mathbf{c} / \rightarrow / \mathbf{c} /$.

$C A_{39}$ l'alternance $/ \mathrm{g} / \sim / \mathrm{g} /$ qui se produit dans la structure du radical flexionnel des lexèmes de différentes classes grammaticales du type GRAUR, GAFĂ, GRAD, GRABNIC, A GUSTA, en se réalisant aussi comme l'alternance directionnelle $\boldsymbol{d} \boldsymbol{C A}_{39} / \mathbf{g} / \rightarrow / g /$.

$C A_{40}$ l'alternance $/ \mathrm{g} / \sim / \mathrm{d} /$ qui se produit

[1] dans la structure du radical flexionnel des lexèmes de différentes classes grammaticales du type COVRIG, BEŞLEAGĂ, ALGĂ, VERIGĂ, CÂRLIG, VITREG, LUNG, A CÂŞTIGA, A RUMEGA, A ADĂUGA, A LEGĂNA, en

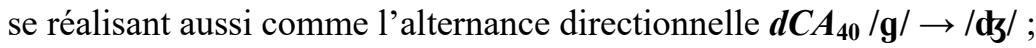

[2] dans la structure du radical flexionnel des verbes A SUGE, A FRIGE, A ÎNFIGE, tout en faisant partie de l'alternance trimembre $C A_{2} / \mathrm{g} / \sim$ $/ \mathrm{d} / \sim / \mathrm{p} /$

[3] dans la structure du radical flexionnel des verbes du type A TRAGE, A STINGE, A ALEGE, A FRÂNGE, A SPARGE, tout en faisant partie de l'alternance trimembre $C A_{4} / \mathrm{g} / \sim / \mathrm{d} / \sim \varnothing$.

$C A_{41}$ l'alternance $/ \mathrm{g} / \sim / \mathrm{f} /$ qui se produit dans la structure du radical flexionnel des verbes du type A DIVAGA, A INTEROGA, A NAVIGA, en se réalisant aussi comme l'alternance directionnelle $\boldsymbol{d} \boldsymbol{C} \boldsymbol{A}_{41} / \mathbf{g} / \rightarrow / \mathbf{J} /$.

$C A_{42}$ l'alternance $/ \mathrm{k} / \sim / \mathrm{k} /$ qui se produit dans la structure du radical flexionnel des lexèmes de différentes classes grammaticales du type CODRU, CRIMĂ, CLOCOT, COMOD, CARE, A CULTIVA, en se réalisant aussi comme l'alternance directionnelle $\boldsymbol{d} \boldsymbol{C A}_{\mathbf{4 2}} / \mathbf{k} / \rightarrow / \mathbf{k}$.

$C A_{43}$ l'alternance $/ \mathrm{k} / \sim / \mathrm{g} /$ qui se produit

[1] dans la structure du radical flexionnel des lexèmes de différentes classes grammaticales du type COPAC, DUCĂ, VLĂDICĂ, ŢĂRANCĂ, NUCĂ, IBRIC, SĂRAC, ADÂNC, A ARUNCA, A PROVOCA, A FERMECA, en

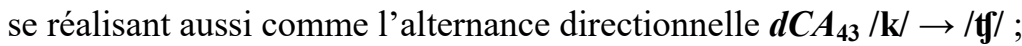

[2] dans la structure du radical flexionnel des verbes A COACE, A RĂSCOACE, A RECOACE, tout en faisant partie de l'alternance trimembre $C A_{3} / \mathrm{k} / \sim$ $/ \mathrm{g} / \sim / \mathrm{p} /$

[3] dans la structure du radical flexionnel des verbes du type A TOARCE, A FACE, A ADUCE, tout en faisant partie de l'alternance trimembre $C A_{5} / \mathrm{k} / \sim / \mathrm{g} / \sim \varnothing$.

$C A_{44}$ l'alternance $/ \mathrm{k} / \sim / \mathrm{c} /$ qui se produit dans la structure du radical flexionnel des verbes du type A PARCA, A BLOCA, A DEFECA, en se réalisant aussi comme l'alternance directionnelle $\boldsymbol{d C A _ { 4 4 }} / \mathbf{k} / \rightarrow / \mathbf{c} /$.

III-11. les alternances bimembres monophonémiques réductrices :

$C A_{45}$ l'alternance $/ \mathrm{n} / \sim \varnothing$ qui se produit dans la structure du radical flexionnel des verbes du type A ŢINE, A VENI, A MÂNEA, en se réalisant aussi comme

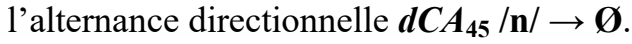


$C A_{46}$ l'alternance $/ 1 / \sim \varnothing$ qui se produit dans la structure du radical flexionnel des substantifs du type COPIL, PIELE, des adjectifs du type DESTUL, MOALE, FRUMUŞEL, MIŞEL, et des pronoms du type EL, CEL, ACEL, en se réalisant aussi comme l'alternance directionnelle $\boldsymbol{d C \boldsymbol { A } _ { 4 6 }} / \mathbf{l} / \rightarrow \boldsymbol{\varnothing}$.

$C A_{47}$ l'alternance $/ \mathrm{r} / \sim \varnothing$ qui se produit dans la structure du radical flexionnel du verbe A PIERI, en se réalisant aussi comme l'alternance directionnelle

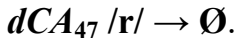

$C A_{48}$ l'alternance $/ \mathrm{b} / \sim \varnothing$ qui se produit dans la structure du radical flexionnel des verbes du type A FIERBE, en se réalisant aussi comme l'alternance

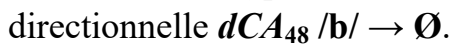

\section{SYSTÈME DE MORPHONÈMES}

Le modèle suivant du système morphonologique consiste en un tout fermé de morphonèmes - unités du second degré d'abstraction. Un morphonème est compris ici comme un ensemble de (deux, trois ou quatre) phonèmes qui alternent. Le statut d'un morphonème est déterminé par deux conditions de base qui doivent être remplies par l'alternance donnée et ses membres constitutifs (phonèmes alternants). Premièrement, l'alternance doit être définie comme directionnelle, c'est-à-dire se produisant dans la direction du membre morphologiquement non-marqué au membre morphologiquement marqué (ou du membre le moins marqué au membre le plus marqué). Deuxièmement, chaque membre d'une telle alternance doit apparaître dans ses propres contextes caractéristiques, strictement déterminés, qui n'acceptent pas d'autre phonème appartenant à la même classe. Le morphonème est représenté par le membre non-marqué (ou le moins marqué) de l'alternance.

Le système de morphonèmes se base d'abord sur les relations d'opposition qualitative, puis quantitative, et enfin purement distributionnelle. Les oppositions qualitatives se limitent ici au statut phonologique des membres alternants : "nonconsonantique $\neq$ consonantique $»$ et $«$ non-consonantique non-transitoire (vocalique) $\neq$ non-consonantique transitoire $»$. Les oppositions quantitatives portent, d'une part, sur le degré de complexité phonologique du membre alternant : " non-monophonémique (biphonémique) $\neq$ monophonémique », et, d'autre part, sur le nombre de membres de l'alternance donnée : « monomembre $\neq$ bimembre $\neq$ trimembre $\neq$ quadrimembre ». Les oppositions du type distributionnel résultent directement des contextes caractéristiques dans lesquels apparaissent les membres alternants appartenant à la même classe phonologique ${ }^{5}$.

Les relations d'opposition choisies, employées conformément à la hiérarchie décrite ci-dessus, conduisent à un total de 117 morphonèmes, dont 56 du type

\footnotetext{
${ }^{5}$ C'est sur ce critère que reposent, dans le premier modèle, les alternances à la même direction, qui ont été isolées comme différentes bien qu'elles soient constituées des membres identiques.
} 
consonantique et 61 du type non-consonantique. Parmi les 61 non-consonantiques il y a 4 morphonèmes transitoires et 57 morphonèmes vocaliques (54 monophonémiques, 3 biphonémiques). Parmi les 56 consonantiques il y a 50 morphonèmes monophonémiques ( 7 semi-ouverts, 13 constrictifs, 8 affriquées, 22 occlusifs) et 6 morphonèmes biphonémiques. Cet ensemble est complété par le zéro morphonémique, réalisé par le $\varnothing$ morphologique et phonologique dans des contextes du type : cola $\varnothing$ /kolaØ/, paciuliØ/patfuliØ/, bejØ/bezØ/.

Voici le tableau complet des morphonèmes avec leurs alternances directionnelles définitoires :

1.1. non-consonantiques non-transitoires non-monophonémiques

- non-antérieurs

$\left|\widehat{\mathrm{oa}}_{1}\right|: d V A_{1.2} / \widehat{\mathrm{oa}} / \rightarrow\{/ \mathrm{o} /, / \mathrm{u} /\}$

$\left|\widehat{o a}_{2}\right|: d V A_{9.3} / \widehat{\mathrm{oa}} / \rightarrow / \mathrm{o} /$

- antérieur

$\left|\widehat{e a}_{1}\right|: d V A_{8.4} / \widehat{e a} / \rightarrow / \mathrm{e} /$

1.2. non-consonantiques non-transitoires monophonémiques

- non-hauts non-bas non-antérieurs nonpostérieurs

$$
\begin{aligned}
& \left|\partial_{0}\right|: d V A_{23} / \mathrm{\partial} / \rightarrow / \mathrm{\partial} / \\
& \left|\partial_{1}\right|: d V A_{5.1} / \mathrm{\partial} / \rightarrow\{/ \mathrm{e} /, / \mathrm{a} /\} \\
& \left|\partial_{2}\right|: d V A_{2.2} / \mathrm{\partial} / \rightarrow\{/ \mathrm{e} /, / \mathrm{ea} /\} \\
& \left|\partial_{3}\right|: d V A_{24.1}(/ \mathrm{\partial} / \rightarrow / \mathrm{e} /)_{1} \\
& \left|\partial_{4}\right|: d V A_{24.2}(/ \mathrm{\partial} / \rightarrow / \mathrm{e} /)_{2} \\
& \left|\partial_{5}\right|: d V A_{24.3}(/ \mathrm{\partial} / \rightarrow / \mathrm{e} /)_{3} \\
& \left|\partial_{6}\right|: d V A_{18.4}(/ \mathrm{\partial} / \rightarrow / \mathrm{a} /)_{1} \\
& \left|\partial_{7}\right|: d V A_{18.5}(/ \mathrm{\partial} / \rightarrow / \mathrm{a} /)_{2} \\
& \left|\partial_{8}\right|: d V A_{18.6}(/ \mathrm{\partial} / \rightarrow / \mathrm{a} /)_{3} \\
& \left|\partial_{9}\right|: d V A_{30.1}(/ \mathrm{\partial} / \rightarrow \varnothing)_{1} \\
& \left|\partial_{10}\right|: d V A_{30.2}(/ \mathrm{\partial} / \rightarrow \emptyset)_{2}
\end{aligned}
$$

- non-hauts non-bas non-antérieurs postérieurs

$$
\begin{aligned}
& \left|\mathrm{o}_{0}\right|: d V A_{21} / \mathrm{o} / \rightarrow / \mathrm{o} / \\
& \left|\mathrm{o}_{1}\right|: d V A_{22} / \mathrm{o} / \rightarrow / \mathrm{a} / \\
& \left|\mathrm{o}_{2}\right|: d V A_{9.1}(/ \mathrm{o} / \rightarrow / \overline{\mathrm{oa}} /)_{1} \\
& \left|\mathrm{o}_{3}\right|: d V A_{9.2}(/ \mathrm{o} / \rightarrow / \overline{\mathrm{oa}} /)_{2} \\
& \left|\mathrm{o}_{4}\right|: d V A_{29} / \mathrm{o} / \rightarrow \varnothing \\
& \left|\mathrm{o}_{5}\right|: d T A_{3} / \mathrm{o} / \rightarrow / \mathrm{w} /
\end{aligned}
$$

- non-hauts non-bas antérieurs $\left|\mathrm{e}_{0}\right|: d V A_{19} / \mathrm{e} / \rightarrow / \mathrm{e} /$ $\left|\mathrm{e}_{1}\right|: d V A_{5.2} / \mathrm{e} / \rightarrow\{/ \mathrm{o} /, / \mathrm{a} /\}$ $\left|\mathrm{e}_{2}\right|: d V A_{2.1} / \mathrm{e} / \rightarrow\{/ \overline{\mathrm{ea}} /, / \mathrm{\partial} /\}$ $\left|\mathrm{e}_{3}\right|: d V A_{3} / \mathrm{e} / \rightarrow\{$ /ea $/, \varnothing\}$ $\left|\mathrm{e}_{4}\right|: d V A_{24.4} / \mathrm{e} / \rightarrow / \mathrm{a} /$ $\left|\mathrm{e}_{5}\right|: d V A_{17.3}(/ \mathrm{e} / \rightarrow / \mathrm{a} /)_{1}$ $\left|\mathrm{e}_{6}\right|: d V A_{17.4}(/ \mathrm{e} / \rightarrow / \mathrm{a} /)_{2}$ $\left|\mathrm{e}_{7}\right|: d V A_{17.5}(/ \mathrm{e} / \rightarrow / \mathrm{a} /)_{3}$ $\left|\mathrm{e}_{8}\right|: d V A_{8.1}(/ \mathrm{e} / \rightarrow \widehat{\mathrm{ea}} /)_{1}$ $\left|\mathrm{e}_{9}\right|: d V A_{8.2}(/ \mathrm{e} / \rightarrow \widehat{\mathrm{ea}} /)_{2}$ $\left|\mathrm{e}_{10}\right|: d V A_{8.3}(/ \mathrm{e} / \rightarrow \widehat{\mathrm{ea}} /)_{3}$ $\left|\mathrm{e}_{11}\right|: d V A_{28.1}(/ \mathrm{e} / \rightarrow \varnothing)_{1}$ $\left|\mathrm{e}_{12}\right|: d V A_{28.2}(/ \mathrm{e} / \rightarrow \varnothing)_{2}$ $\left|\mathrm{e}_{13}\right|: d V A_{20} / \mathrm{e} / \rightarrow / \mathrm{i} /$

- non-hauts bas

$\left|\mathrm{a}_{0}\right|: d V A_{16} / \mathrm{a} / \rightarrow / \mathrm{a} /$ $\left|\mathrm{a}_{1}\right|: d V A_{7} / \mathrm{a} / \rightarrow\{/ \mathrm{a} /, \varnothing\}$ $\left|\mathrm{a}_{2}\right|: d V A_{6} / \mathrm{a} / \rightarrow\{/ \mathrm{e} /, \varnothing\}$ $\left|\mathrm{a}_{3}\right|: d V A_{17.1}(/ \mathrm{a} / \rightarrow / \mathrm{e} /)_{1}$ $\left|\mathrm{a}_{4}\right|: d V A_{17.2}(/ \mathrm{a} / \rightarrow / \mathrm{e} /)_{2}$ $\left|\mathrm{a}_{5}\right|: d V A_{18.1}(/ \mathrm{a} / \rightarrow / \mathrm{o} /)_{1}$ $\left|\mathrm{a}_{6}\right|: d V A_{18.2}(/ \mathrm{a} / \rightarrow / \mathrm{a} /)_{2}$ $\left|\mathrm{a}_{7}\right|: d V A_{18.3}(/ \mathrm{a} / \rightarrow / \mathrm{o} /)_{3}$

- hauts non-antérieurs non-postérieurs

$\left|\dot{\mathbf{i}}_{0}\right|: d V A_{13} / \mathbf{i} / \rightarrow / \mathbf{i} /$

$\left|\mathrm{i}_{1}\right|: d V A_{4} / \mathrm{i} / \rightarrow\{/ \mathrm{a} /, / \mathrm{a} /\}$

$\left|\mathbf{i}_{2}\right|: d V A_{14} / \mathbf{i} / \rightarrow / \mathrm{a} /$

$\left|\mathbf{i}_{3}\right|: d V A_{27.1}(/ \mathbf{i} / \rightarrow \varnothing)_{1}$

$\left|\mathbf{i}_{4}\right|: d V A_{27.2}(/ \mathbf{i} / \rightarrow \emptyset)_{2}$

$\left|\mathbf{i}_{5}\right|: d V A_{15.1} / \mathbf{i} / \rightarrow / \mathbf{i} /$ 
- hauts non-antérieurs postérieurs

$\left|\mathrm{u}_{0}\right|: d V A_{11} / \mathrm{u} / \rightarrow / \mathrm{u} /$

$\left|\mathrm{u}_{1}\right|: d V A_{1.1} / \mathrm{u} / \rightarrow\{/ \mathrm{o} /, / \mathrm{oa} /\}$

$\left|\mathrm{u}_{2}\right|: d V A_{12} / \mathrm{u} / \rightarrow / \mathrm{a} /$

$\left|\mathrm{u}_{3}\right|: d V A_{26} / \mathrm{u} / \rightarrow \varnothing$

$\left|\mathrm{u}_{4}\right|: d T A_{2.1} / \mathrm{u} / \rightarrow / \mathrm{w} /$

- hauts antérieurs

$\left|\mathrm{i}_{0}\right|: d V A_{10} / \mathrm{i} / \rightarrow / \mathrm{i} /$

$\left|\mathrm{i}_{1}\right|: d V A_{25} / \mathrm{i} / \rightarrow \varnothing$

$\left|\mathbf{i}_{2}\right|: d V A_{15.2} / \mathbf{i} / \rightarrow / \mathbf{i} /$

$\left|\mathrm{i}_{3}\right|: d T A_{1.1} / \mathrm{i} / \rightarrow / \mathrm{j} /$

2.1. non-consonantiques transitoires non-monophonémiques

2.2. non-consonantiques transitoires monophonémiques

- non-antérieurs

$\left|\mathrm{w}_{0}\right|: d T A_{5} / \mathrm{w} / \rightarrow / \mathrm{w} /$

$\left|\mathrm{w}_{1}\right|: d T A_{2.2} / \mathrm{w} / \rightarrow / \mathrm{u} /$

- antérieurs

$\left|\mathrm{j}_{0}\right|: d T A_{4} / \mathrm{j} / \rightarrow / \mathrm{j} /$

$\left|\mathrm{j}_{1}\right|: d T A_{1.2} / \mathrm{j} / \rightarrow / \mathrm{i} /$

3.1. consonantiques nonmonophonémiques

- non-palataux + non-apicaux

$\left|\widehat{\mathrm{sk}}_{1}\right|: d C A_{9} / \widehat{\mathrm{sk}} / \rightarrow \widehat{\mathrm{sg}} /$

$\left|\mathrm{sk}_{2}\right|: d C A_{10.1} / \mathrm{sk} / \rightarrow / \widetilde{\mathrm{ft}} /$

- non-palatal + apical

$\left|\widehat{s t}_{1}\right|: d C A_{8} / \widehat{\mathrm{st} t} \rightarrow / \widehat{\mathrm{ft} t} /$

- palataux + non-apicaux

$|\widehat{\sqrt{k}}|: d C A_{11} / \widehat{\sqrt{k}} / \rightarrow / \widetilde{J g} /$

$|\widetilde{\sqrt{ }} 2|: d C A_{12} / \widetilde{\widehat{k}} / \rightarrow / \widetilde{\mathrm{t} t}$

- palatal + apical

$\left|\widehat{\int \mathrm{f}_{1}}\right|: d C A_{10.2} / \widetilde{\mathrm{Jt}} / \rightarrow / \widehat{\mathrm{sk}} /$

3.2. consonantiques monophonémiques

3.2.1. non-semi-ouverts non-constrictifs

- non-labiaux non-apicaux non-palataux non-sonores $\left|\mathrm{k}_{0}\right|: d C A_{42} / \mathrm{k} / \rightarrow / \mathrm{k} /$

$\left|\mathrm{k}_{1}\right|: d C A_{43} / \mathrm{k} / \rightarrow / \mathrm{g} /$

$\left|\mathrm{k}_{2}\right|: d C A_{44} / \mathrm{k} / \rightarrow / \mathrm{c} /$

- non-labiaux non-apicaux non-palataux sonores

$\left|\mathrm{g}_{0}\right|: d C A_{39} / \mathrm{g} / \rightarrow / \mathrm{g} /$

$\left|\mathrm{g}_{1}\right|: d C A_{40} / \mathrm{g} / \rightarrow / \mathrm{d}_{\mathrm{d}} /$

$\left|\mathrm{g}_{2}\right|: d C A_{41} / \mathrm{g} / \rightarrow / \mathrm{J} /$

- non-labial non-apical palatal nonsonore

$\left|\mathrm{c}_{0}\right|: d C A_{38} / \mathrm{c} / \rightarrow / \mathrm{c} /$

- non-labial non-apical palatal sonore

$\left|\mathrm{J}_{0}\right|: d C A_{37} / \mathrm{J} / \rightarrow / \mathrm{J} /$

- non-labiaux apicaux non-sonores

$\left|\mathrm{t}_{0}\right|: d C A_{35} / \mathrm{t} / \rightarrow / \mathrm{t} /$

$\left|\mathrm{t}_{1}\right|: d C A_{36.1}(/ \mathrm{t} / \rightarrow / \mathrm{ts} /)_{1}$

$\left|\mathrm{t}_{2}\right|: d C A_{36.2}(/ \mathrm{t} / \rightarrow / \mathrm{ts} /)_{2}$

$\left|\mathrm{t}_{3}\right|: d C A_{7} / \mathrm{t} / \rightarrow\{/ \mathrm{ts} /, \varnothing\}$

- non-labiaux apicaux sonores

$\left|\mathrm{d}_{0}\right|: d C A_{32} / \mathrm{d} / \rightarrow / \mathrm{d} /$

$\left|\mathrm{d}_{1}\right|: d C A_{33.1}(/ \mathrm{d} / \rightarrow / \mathrm{z} /)_{1}$

$\left|\mathrm{d}_{2}\right|: d C A_{33.2}(/ \mathrm{d} / \rightarrow / \mathrm{z} /)_{2}$

$\left|\mathrm{d}_{3}\right|: d C A_{33.3}(/ \mathrm{d} / \rightarrow / \mathrm{z} /)_{3}$

$\left|\mathrm{d}_{4}\right|: d C A_{34} / \mathrm{d} / \rightarrow / 3 /$

$\left|\mathrm{d}_{5}\right|: d C A_{6} / \mathrm{d} / \rightarrow\{/ \mathrm{z} /, \varnothing\}$

$\left|\mathrm{d}_{6}\right|: d C A_{1} / \mathrm{d} / \rightarrow\{/ \mathrm{z} /, / \mathrm{g} /, \varnothing\}$

- labial non-sonore

$\left|\mathrm{p}_{0}\right|: d C A_{31} / \mathrm{p} / \rightarrow / \mathrm{p} /$

- labiaux sonores

$\left|\mathrm{b}_{0}\right|: d C A_{30} / \mathrm{b} / \rightarrow / \mathrm{b} /$

$\left|\mathrm{b}_{1}\right|: d C A_{48} / \mathrm{b} / \rightarrow \varnothing$

3.2.2. non-semi-ouverts constrictifs non-occlusifs

- non-labiaux non-apicaux non-palataux

$\left|\mathrm{x}_{0}\right|: d C A_{25} / \mathrm{x} / \rightarrow / \mathrm{x} /$

$\left|\mathrm{x}_{1}\right|: d C A_{26} / \mathrm{x} / \rightarrow / \mathrm{S} /$

- non-labiaux non-apicaux palataux non-sonores

$\left|\int_{0}\right|: d C A_{24} / \mathrm{J} / \rightarrow / \mathrm{J} /$

$\left|\mathrm{S}_{1}\right|: d C A_{22.3} / \mathrm{g} / \rightarrow / \mathrm{s} /$ 
- non-labial non-apical palatal sonore $\left|3_{0}\right|: d C A_{23} / 3 / \rightarrow / 3 /$

- non-labiaux apicaux non-sonores

$\left|\mathrm{s}_{0}\right|: d C A_{21} / \mathrm{s} / \rightarrow / \mathrm{s} /$

$\left|\mathrm{s}_{1}\right|: d C A_{22.1}(/ \mathrm{s} / \rightarrow / \mathrm{J} /)_{1}$

$\left|\mathrm{s}_{2}\right|: d A C_{22.2}(/ \mathrm{s} / \rightarrow / \mathrm{J} /)_{2}$

- non-labiaux apicaux sonores

$\left|\mathrm{z}_{0}\right|: d C A_{19} / \mathrm{z} / \rightarrow / \mathrm{z} /$

$\left|\mathrm{z}_{1}\right|: d C A_{33.4} / \mathrm{z} / \rightarrow / \mathrm{d} /$

$\left|\mathrm{z}_{2}\right|: d C A_{20} / \mathrm{z} / \rightarrow / \mathrm{z} /$

- labial non-sonore

$\left|\mathrm{f}_{0}\right|: d C A_{18} / \mathrm{f} / \rightarrow / \mathrm{f} /$

- labial sonore

$\left|\mathrm{v}_{0}\right|: d C A_{17} / \mathrm{v} / \rightarrow / \mathrm{v} /$

3.2.3. non-semi-ouverts constrictifs occlusifs

- non-apicaux non-sonores

$\left|\mathfrak{f}_{0}\right|: d C A_{29} / \mathfrak{g} / \rightarrow / \mathfrak{g} /$

$\left|\mathrm{f}_{1}\right|: d C A_{3} / \mathrm{g} / \rightarrow\{/ \mathrm{k} /, / \mathrm{p} /\}$

$\left|\mathrm{t}_{2}\right|: d C A_{5} / \mathrm{t} / \rightarrow\{/ \mathrm{k} /, \varnothing\}$
- non-apicaux sonores

$\left|\mathrm{d}_{0}\right|: d C A_{28} / \mathrm{d} / \rightarrow / \mathrm{d} /$

$\left|\mathrm{d}_{1}\right|: d C A_{2} / \mathrm{d} / \rightarrow\{/ \mathrm{g} /, / \mathrm{p} /\}$

$\left|\mathrm{d}_{2}\right|: d C A_{4} / \mathrm{d} / \rightarrow\{/ \mathrm{g} /, \varnothing\}$

- apicaux

$\left|\mathrm{ts}_{0}\right|: d C A_{27} / \mathrm{ts} / \rightarrow / \mathrm{ts} /$

$\left|\mathrm{ts}_{1}\right|: d C A_{36.3} / \mathrm{ts} / \rightarrow / \mathrm{t} /$

3.2.4. semi-ouverts

- non-nasaux non-latéraux

$\left|\mathrm{r}_{0}\right|: d C A_{16} / \mathrm{r} / \rightarrow / \mathrm{r} /$

$\left|\mathrm{r}_{1}\right|: d C A_{47} / \mathrm{r} / \rightarrow \varnothing$

- non-nasaux latéraux

$\left|1_{0}\right|: d C A_{15} / 1 / \rightarrow / 1 /$

$\left|1_{1}\right|: d C A_{46} / 1 / \rightarrow \varnothing$

- nasaux non-labiaux

$\left|\mathrm{n}_{0}\right|: d C A_{14} / \mathrm{n} / \rightarrow / \mathrm{n} /$

$\left|\mathrm{n}_{1}\right|: d C A_{45} / \mathrm{n} / \rightarrow \varnothing$

- nasal labial

$\left|\mathrm{m}_{0}\right|: d C A_{13} / \mathrm{m} / \rightarrow / \mathrm{m} /$

\section{REMARQUES FINALES}

Les deux modèles du système morphonologique roumain présentés plus haut sont à certains égards des conceptions concurrentes, bien que le système de morphonèmes puisse être dérivé directement du système d'alternances, comme cela a été démontré et entrepris ici. Premièrement, l'un et l'autre modèle rendent possible l'élaboration effective d'une description exhaustive des phénomènes morphonologiques qui caractérisent le roumain. Deuxièmement, les deux modèles se prêtent assez facilement aux complètements nécessaires par des éléments d'importance non seulement marginale. Cependant, seul le système se rapportant au concept de morphonème peut être sans doute plus largement appliqué. Définies à l'aide des relations d'opposition, les unités morphonémiques, avec leurs représentations simples, constituent un excellent outil pour toute analyse morphologique, soit-elle flexionnelle, dérivationnelle ou morphotactique. 


\section{BIBLIOGRAPHIE}

Avram, A. (2013). Contribuţii la morfonologia limbii române. Bucureşti : Editura Academiei Române.

Cychnerski, T. (2001). Morfonologia limbii române. I. Paradigmatica. O abordare teoretică pe baza materialului verbal. In Studia Romanica Posnaniensia. XXVIII, pp. 17-22.

Cychnerski, T. (2003). Morfonologia limbii române. II. Sintagmatica. O abordare teoretică pe baza materialului verbal. In Studia Romanica Posnaniensia. XXIX, pp. 139-148.

Guţu Romalo, V. (1985). Morfologia. In I. Coteanu (ed.). Limba română contemporană. Fonetica. Fonologia. Morfologia. Bucureşti.

Harris, Z.S. (1951). Methods in Structural Linguistics. Chicago: The University of Chicago Press. Hockett, Ch.F. (1958). A Course in modern linguistics. New York: The Macmillan Company. Kilbury, J. (1976). The development of morphophonemic theory. Amsterdam: John Benjamins. Vasiliu, E. (1965). Fonologia limbii române. Bucureşti : Editura Ştiinţifică.

Vasiliu, E. (1985). Fonologia. In I. Coteanu (ed.). Limba română contemporană. Fonetica. Fonologia. Morfologia. Bucureşti. 